\title{
Role of Wiener chaos expansion in modelling randomness for groundwater contamination flow
}

\author{
$\underline{\text { P. Tiwari }}^{\mathrm{a}}$, D. Kulasiri ${ }^{\mathrm{a}, \mathrm{b}}$ and S. Samarasinghe ${ }^{\mathrm{b}, \mathrm{c}}$ \\ ${ }^{a}$ Centre for Advanced Computational Solutions (C-fACS), Lincoln University, Christchurch, New Zealand \\ ${ }^{b}$ Complex Systems, Big Data and Informatics Initiative (CSBII), Lincoln University, New Zealand \\ ${ }^{c}$ Department of Environmental Management, Lincoln University, New Zealand \\ Email: Parul.Tiwari@lincolnuni.ac.nz
}

\begin{abstract}
Pertinent mathematical modelling plays pivot role in making groundwater protection and reclamation policies. Uncertain parameters and several basic phenomena in almost all branches of engineering and science can be modelled efficiently with the help of Stochastic Partial Differential Equations (SPDEs) and their behaviour can be interpreted more accurately. The intent of the present study is to use an efficient numerical approach based on Wiener chaos expansion to understand the stochastic nature of variables associated with groundwater flow. First and second order moments of concentration profile are calculated and plotted graphically. Obtained results are in good aggrement with those available in existing literature.
\end{abstract}

Keywords: $\quad$ Stochastic partial differential equations, Wiener chaos, hermite polynomials, stochastic simulation, brownian motion 


\section{INTRODUCTION}

"We're all stumbling in the dark, and that makes for some pretty interesting collisions, where the fog is thickest, begin"

- Marty Rubin

Uncertainty quantification seeks to address the problems associated with randomness in real world systems and their probabilistic behaviour (Chernatynskiy, Phillpot, \& LeSar, 2013). It is innate part of the real world. It is almost unexpected to get exactly the same outcome for two physical experimentsasmany significant inputs may be unknown or exorbitant. Uncertainty affects almost all aspects of engineering modelling and design (Tegegne, Kim, Seo, \& Kim, 2019; Ye \& Zhu, 2018). By acute understanding and gauging the sources of uncertainty, a researcher can make better decisions with known levels of confidence. Uncertainty that comes from the lack of knowledge called epistemic uncertainty and can be reduced by gathering more information and having more measurements. Another type of uncertainty that is inherent in the process called aleatory uncertainty and cannot be reduced. These uncertainties are characterized by probability distribution. Since it is hard to get exact information, uncertainties may be best modelled by appropriate assumptions and with adequate information available. Thus to develop a good mathematical model to account the fluctuations of an uncertain quantity is to to treat it as a stochastic quantity in a suitable space of stochastic distribution and to use suitable stochastic model. The validity of these models may be answered by comparing their speculations with reality and certified experimental data (Christakos, Hristopulos, \& Miller, 1995).

Though parameter estimation and uncertainty quantification are two key components of modern science-based forecasting, predictions can lead to more accurate results when governing equations model the physics of the problem. One of the standard example is to solve a moving boundary value problem for the unknown pressure of a fluid flow in which permeability of the medium is unpredictable and varies unevenly. When modelling Groundwater contamination (Natarajan \& Kumar, 2018) results show that these tasks must cope with many degrees of freedom and large datasets. In groundwater hydrology, permeability is a random parameter that is inherent in the environment surrounding the system. Appropriate mathematical tool to deal with these uncertainties is to formulate the system as an SPDE. Stochastic permeability of a heterogeneous and isotropic medium is modelled as smoothed positive noise process by (H. Holden, Oksendal, Uboe, \& Zhang, 1996) and solution of governing system of equations is obtained in a suitable space of stochastic distributions known as Kondratiev space (Kondratiev, Leukert, \& Streit, 1996).

Numerous dedicated researchers and experts in hydrology (Helge Holden, Lindstrøm, Øksendal, Ubøe, \& Zhang, 1993; Serrano \& Unny, 1987; Xu et al., 2018) accelerate the research on SPDEs. Stochastic differential equations are being used for modelling hydrological systems for a long time (Gupta, Bhattacharya, \& Sposito, 1981) and still the researchers are actively involved to find the optimal solution strategies to deal with irregularities (Asmuth \& Bierkens, 2005; D'Odorico, Laio, \& Ridolfi, 2005; Peterson \& Western, 2014). These SPDEs are either driven by space-time Brownian motion (Kalpinelli, Frangos, \& Yannacopoulos, 2011; Kulasiri, 2013) or more generally, space-time Levy process (Arne, Oksendal, \& Frank, 2004; Chen, Rozovskii, \& A Shu, 2019) and solved analytically as well as numerically in several monographs and research articles. Brownian motion is defined as the irregular movement of particles suspended in a fluid. It is a continuous adapted process defined on some probability space. The motion identified is due to the statistical fluctuations. A stochastic process $B=\{B(t): t \geq 0\}$ having continuous random path is called standard Brownian motion if $B(t, \omega)=0$ for a realization $\omega$ at $t=0$ and the increments of Brownian motion are independent and normally distributed with mean zero and variance $\left(t_{i+1}-t_{i}\right)$ for all $i \geq 0$. The stochastic variation of $B(t, \omega)$ at time $t$ is determined by Gaussian probability distribution which has a zero mean for all values of $t$. Whereas a stochastic process $X=\{X(t): t \geq 0\}$ is said to be Lévy process if $X(0)=0$ almost surely and its increments over non-overlapping time intervals are independent. In Lévy process for any $t_{i}<t_{i+1}, X\left(t_{i+1}\right)-X\left(t_{i}\right)$ is equal in distribution to $X_{t_{i+1}-t_{i}}$. Detailed theory of SPDEs and the related concepts can be found in (Da Prato \& Zabczyk, 2014; H. Holden et al., 1996; Z. a. Zhang, 2017).

Speedy development of fast computer processors enable the researchers to make accurate predictions for more real world systems and competitive frontier of engineering design has moved on to quickly predicting the behaviour of these systems when subjected to uncertain inputs. In SPDEs, uncertain quantities can be characterized using probability theory either using random sampling by Monte Carlo (MCMC) based methods or by polynomial chaos methods such as Wiener-Hermite chaos (Luo, 2006; Z. Zhang, Rozovskii, Tretyakov, \& Karniadakis, 2012). 
In the present work, our prime focus is on modelling the stochastic quantity in suitable mathematical frame work and developing efficient numerical analogy of computationally expensive numerical models. In this paper, we consider groundwater contamination flow governed by standard advection dispersion equation. Wiener chaos expansion (WCE) is used to characterise the random fluctuations in the stochastic quantity. Wiener chaos or simply polynomial chaos play an important role in modern probability theory. The fundamental concept of using this method is to develop the solution as part of an infinite series that may converge in Wiener chaos space that contain useful information on the statistical properties of the random process generated by SPDE. Wiener chaos is a non-sampling technique to characterize the evolution of probabilistic irregularity in a dynamical system. The advantage of using this approach lies in the fact that wiener chaos coefficients can be calculated by reducing the uncertainty propagator into lower triangular matrix. The fundamental concept of using this method is to develop the solution as part of an infinite series that may converge in Wiener chaos space. Thus, this technique works as an efficient analytical and theoretical tool to study current and peculiar kind of solutions. The paper is organised as follows:

Section 2 explains the basic concepts and theory of Wiener-Hermite chaos expansion. Development and implementation of the theory is described in section 3 and deals with the proposed algorithm and its numerical implementation to the stochastic partial differential equation. Discussion and numerical results are elucidated in section 4. Finally upshots are given in section 5.

\section{WIENER-CHAOS EXPANSION (WCE)}

Based on Cameron-Martin Theorem (Cameron \& Martin, 1947), the random solution of an SPDE can be represented in terms of an infinite series known as Wiener chaos expansion. This expansion can separate the deterministic effects from the randomness in the random solution. The deterministic coefficients in this expansion are called Wiener chaos coefficients and can be obtained by solving a Wiener chaos propagator, which is a deterministic system and can be solved using standard numerical methods. Unlike in Monte Carlo simulation, there is no need to generate thousands of random numbers to solve an SPDE. The WCE propagator is solved once and based on its Wiener chaos expansion, we can construct the realizations of the random solution. One key point to keep in mind is that we need to design a truncation policy for the Wiener chaos expansion to solve this infinite system of equations. Let us now explain the concept of Wiener chaos expansion mathematically. Given a complete probability space $(\Omega, \vartheta, \mu)$, where $\vartheta$ is the $\sigma$-algebra generated by the set of i.i.d. Gaussian random variables $\left\{\xi_{i}\right\}_{i \geq 1}$ and $\mu$ is a probability measure in $(\Omega, \vartheta)$. Let us denote the set of all multi-indices $\alpha$ by $\mathfrak{I}$ in which $\alpha=\left(\alpha_{1}, \alpha_{2}, \ldots ..\right) \in \mathbb{N}_{0}^{N}$ with only finitely $\alpha_{i} \neq 0$. Also $|\alpha|=\sum_{i \geq 1} \alpha_{i}<\infty$ and $\alpha<\beta$ if $\alpha_{i}<\beta_{i} \forall i \in \mathbb{N}$ $\alpha+\beta:=\left(\alpha_{1}+\beta_{1}, \alpha_{2}+\beta_{2}, \ldots \ldots\right) \forall i \geq 1, \alpha, \beta \in \mathbb{N}_{0}^{N}$

According to Cameron-Martin Theorem (Cameron \& Martin, 1947), any function $f \in L^{2}(\Omega)$ can be uniquely expressed as Wiener Chaos expansion -

$$
f=\sum_{\alpha \in \mathfrak{I}} f_{\alpha} H_{\alpha}
$$

and $\|f\|_{L^{2}(\Omega)}^{2}=\sum_{\alpha \in \mathfrak{I}} f_{\alpha}^{2} \alpha !, f_{\alpha} \in \mathbb{R}$ are the deterministic functions known as $\alpha^{\text {th }}$ Wiener chaos expansion coefficients of function $\downarrow$ and the stochastic term $\left\{H_{\alpha}\right\}_{\alpha \in \Im}$ forms a family of complete orthonormal basis for square integrable space $L^{2}(\Omega)$ and is given as

$$
H_{\alpha}\{\xi\}=\prod_{i \geq 1} H_{\alpha_{i}}\left\{\xi_{i}\right\}
$$

and $\mathrm{E}\left[H_{\alpha} H_{\beta}\right]=\alpha ! \delta_{\alpha \beta}$ in which $H_{n}(\xi)$ are the Hermite polynomials for $n \in \mathbb{N}_{0}, x \in \mathbb{R}$

More details about these polynomials and the orthonormal basis can be found in (H. Holden et al., 1996). 
A stochastic evolution equation with white noise/Brownian motion forcing is written in general form as-

$$
f_{t}=\mathrm{L}(f)+\varsigma(x, t) d W_{t}
$$

Where $\mathrm{L}$ is the linear/nonlinear differential operator in space such as Laplacian operator in case of stochastic advection dispersion equation,

$\varsigma(x, t)$ is the random forcing term and $W_{t}$ is the Brownian motion. Figure (1) shows one sample realization of Brownian motion.

Let us consider the groundwater contamination flow equation, subject to white noise input given by (4)

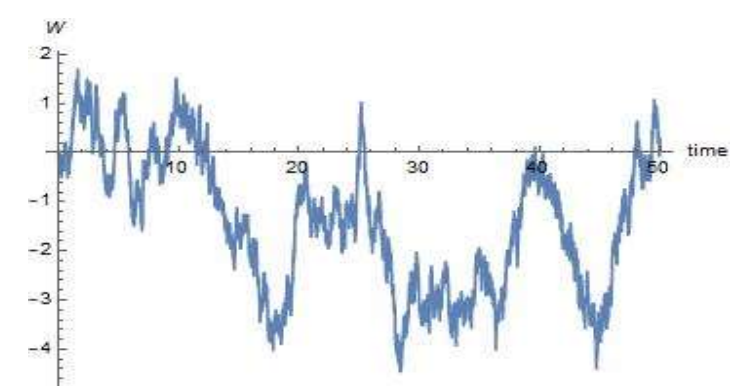

Figure 1. Realization of a sample path of

$d C(x, t)=v \frac{\partial^{2} C(x, t)}{\partial x^{2}} d t+\sigma \frac{\partial C(x, t)}{\partial x} d W(t)$

where $C(x, t)$ represents the concentration of mass transfer, $v$ is the diffusion coefficient and second term on the right hand side of (5) shows the velocity field with white noise forcing. Integral form of (5) is

$$
C(x, t)=C_{0}(x)+v \int_{0}^{t} C_{x x}(x, s) d s+\sigma(x) C_{x}(x, t) W(t)
$$

whereas the deterministic initial condition is taken as $C(x, 0)=C_{0}(x)$.

Polynomial chaos expansion based on Cameron-Martin theorem is applied to develop the numerical solution for (5). Let the solution of this type of equation is given by a random process $C:=C(x, t)$. Computational procedure to find the solution is as follows:

- $\quad$ Express the solution in terms of an infinite series called Wiener-Hermite chaos expansion as in(1)

$$
C(x, t)=\sum_{\alpha \in \mathfrak{I}} C_{\alpha}(x, t) H_{\alpha}, \forall t \leq T
$$

where $C_{\alpha}(x, t)=E\left[C(x, t) H_{\alpha}\right]$, we need to calculate the deterministic functions $C_{\alpha}(x, t)$ known as Wiener chaos coefficients.

- Multiplying both sides of equation (5) by $H_{\alpha}$, taking expectation and using orthogonality of $H_{\alpha}$, we get

$$
C_{\alpha}(x, t)=C_{0}(x) I_{\{\alpha=0\}}+v \int_{0}^{t} C_{x x}(x, s) d s+\sigma(x) C_{x}(x, t) \sum_{i=1}^{\infty} I_{\left\{\alpha_{i}=\delta_{i j}\right\}} B_{i}(s) d s
$$

In which $I_{\{\alpha=0\}}$ is the indicator function which is unity for zero value of $\alpha$ and zero otherwise; $E\left[\xi_{i} \xi_{j}\right]=\delta_{i j}, i \neq j$ and $B_{i}(s)$ are the orthonormal base in $L^{2}(\Omega)$ and can be choosen as trigonometric functions.

- $\quad$ Define the Gaussian random variables $\xi_{i}=\int_{0}^{T} B_{k}(t) d W(t)$ and Hermite polynomials $H_{\alpha}$ for multi-indices $\alpha$. 
- The system of partial differential equations of WCE coeffients is given by (8) and can be written as

$$
\frac{\partial}{\partial t} C_{\alpha}(x, t)=v C_{x x}(x, s)+\sigma(x) \frac{\partial}{\partial x} C_{\alpha}(x, t) \sum_{i=1}^{\infty} I_{\left\{\alpha_{i}=\delta_{i j}\right\}} B_{i}(s)
$$

This is an infinite system of partial differential equations and we truncate it at finite terms for finite Gaussian random variables, say $p$ and order of Hermite polynomials, say, $N$. The truncated WCE is given as

$$
C_{p, N}(x, t)=\sum_{|\alpha| \leq N} C_{\alpha}(x, t) \prod_{i=1}^{p} H_{\alpha_{i}}\left(\xi_{i}\right), \forall t \leq T
$$

Order of truncation error and convergence of the scheme is explained in (Luo, 2006).

- Reconstruct the moments from the solution of deterministic propagator given by (7). Mean and variance of the truncated function are obtained as:

$$
E\left[C_{p, N}(x, t)\right]=C_{0}(x, t) ; \operatorname{var}\left[C_{p, N}(x, t)\right]=\sum_{\alpha \in \mathfrak{I}_{p, N}, \alpha \neq 0}\left|C_{\alpha}(x, t)\right|^{2}
$$

Total number of terms in the truncation is $\frac{p+N !}{p ! N !}$. To avoid so many coefficients in computation, (Luo, 2006) used sparse truncation which reduced the number of WCE coefficients dramatically.

\section{NUMERICAL RESULTS AND DISCUSSION}

For numerical simulation using proposed method, we take $v=0.002, T=0.5$ and $|\sigma| \leq 0.5$. The order of convergence of the method is explained in (Luo, 2006)and error induced due to truncation of the infinite series is $O\left[\sigma(T / P)^{3 / 2}\right]$. It has also been observed that WCE method converges exponentially for polynomial order $N$ and only algebraically for Gaussian random variables $p$.

We considered five Gaussian random variables i.e., $p=5$ and truncate the WCE propagator for $N=1,2$ and 3 . Thus, total number of WCE coefficients are 6,21 and 56 respectively. These numbers can be reduced by using sparse truncation. Figure (1) illustrates the mean obtained using all three cases and compared with the solution obtained by Monte Carlo simulations with $10^{5}$ realisations. It has been observed that the WCE coefficients decay quickly as the order of polynomials increases. As the order of Wiener-Hermite polynomials increased, decay rate of WCE coefficients is high and depend on lengh of interval $\mathrm{T}$ and variability of $\sigma$. Variance solution for Wiener chaos expansion using third order polynomial truncation is plotted in figure (2). Graph shows reasonable agreement of the proposed method with popular method of solution.

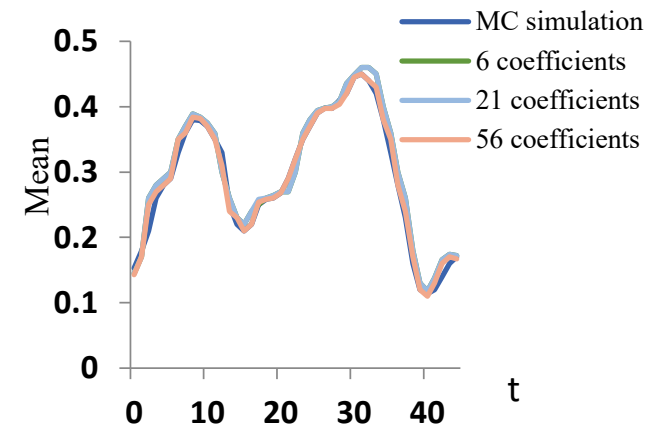

Figure 1. First order statistical moment by WCE method with different WCE coefficients. The first order approximation can not provide accurate results for higher order moments. Moreaccurate results are achieved by using second and higher order approximatins

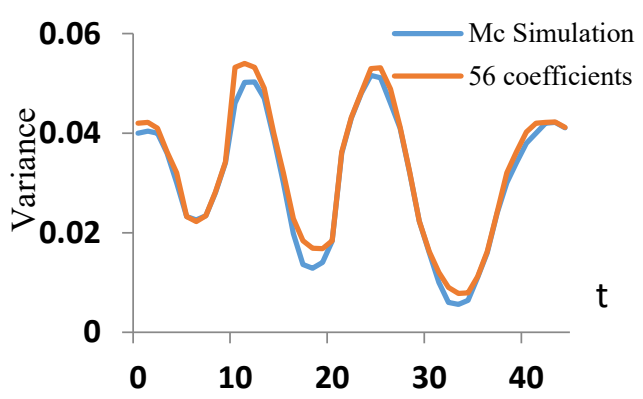

Figure 2. Second order statistical moment by WCE method with third order polynomial approximations compared with Monte Carlo simulations with $10^{5}$ realisations. 


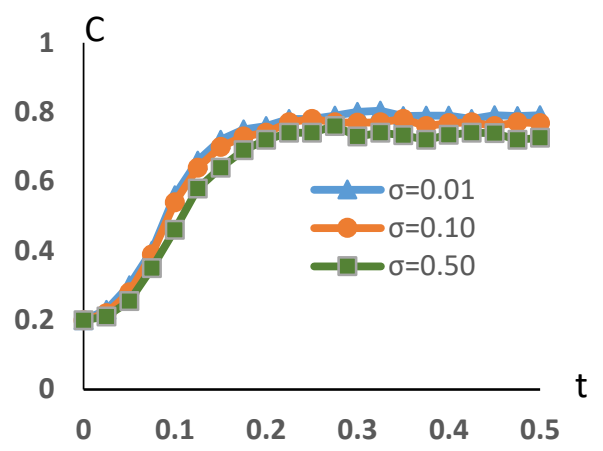

Figure 3. Concentration profile at three different values of $\sigma$. Large perturbation values results in high irregularities in concentration. Asymptotic values have more fluctuations for large values of $\sigma$

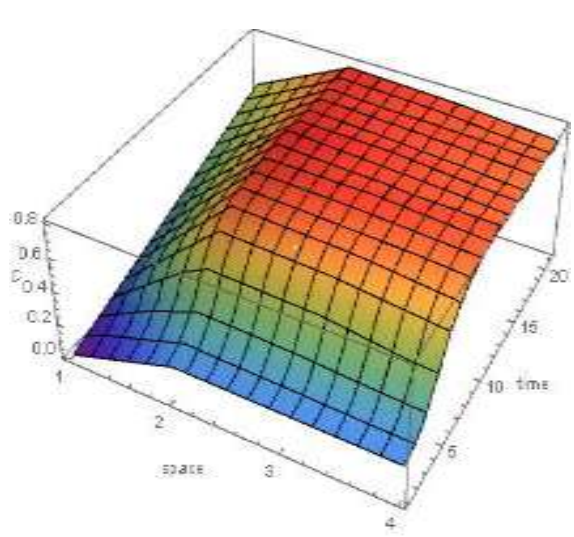

Figure 4. 3D mesh plot for concentration profile at four different positions at different time instances

Concentration profile at $x=0.5$ for different values of $\sigma$ are plotted in figure (3). It is observed from the graph that the converging values of concentration are lower for large purturbations. Figure (4) is the 3D mesh plot for concentration profile at different positions for $\sigma=0.5$ and almost identical results are obtained except smoothening of the asymptotic values.

\section{CONCLUSION}

In this work, Wiener polynomial chaos expansion method is employed to solve one dimensional groundwater flow equation. Using this approach, we got results analogus to the results obtained by Monte Carlo method but with less computational time and efforts. However, the amount of computational effeort depends on the choice of basis and the technique used to solve deterministic system of equations. It is also observed that WCE coefficients diminish with higher rate by increasing $N$ as compared to increasing $p$. Thus, this approach can be used as a substitute to Monte-Carlo type methods under specified boundary and initial conditions.

\section{REFERENCES}

Arne, L., Oksendal, B., \& Frank, P. (2004). Stochastic Partial Differential Equations Driven by Levy Space-Time White Noise. The Annals of Applied Probability, 14(3), 1506-1528.

Asmuth, J., \& Bierkens, M. F. P. (2005). Modeling irregularly spaced residual series as a continuous stochastic process. Water Resources Research, 41. doi:10.1029/2004WR003726

Cameron, R. H., \& Martin, W. T. (1947). The Orthogonal Development of Non-Linear Functionals in Series of Fourier-Hermite Functionals. Annals of Mathematics, 48(2), 385-392. doi: $10.2307 / 1969178$

Chen, T., Rozovskii, B., \& A Shu, C.-W. (2019). Numerical solutions of stochastic PDEs driven by arbitrary type of noise. Stochastics and Partial Differential Equations: Analysis and Computations, 7, 1-39.

Chernatynskiy, A., Phillpot, S. R., \& LeSar, R. (2013). Uncertainty Quantification in Multiscale Simulation of Materials: A Prospective. Annual Review of Materials Research, 43(1), 157-182. doi:10.1146/annurev-matsci-071312-121708

Christakos, G., Hristopulos, D. T., \& Miller, C. T. (1995). Stochastic Diagrammatic Analysis of Groundwater Flow in Heterogeneous Porous Media. Water Resources Research, 31(7), 1687-1703. doi:doi:10.1029/95WR00733

D'Odorico, P., Laio, F., \& Ridolfi, L. (2005). Noise-induced stability in dryland plant ecosystems. Proceedings of the National Academy of Sciences of the United States of America, 102(31), 10819-10822. doi:10.1073/pnas.0502884102 
Tiwari et al., Role of Wiener chaos expansion in modelling randomness for groundwater contamination flow

Da Prato, G., \& Zabczyk, J. (2014). Stochastic Equations in Infinite Dimensions (2 ed.). Cambridge: Cambridge University Press.

Gupta, V. K., Bhattacharya, R. N., \& Sposito, G. (1981). A molecular approach to the foundations of the theory of solute transport in porous media: I. Conservative solutes in homogeneous isotropic saturated media. Journal of Hydrology, 50, 355-370. doi:https://doi.org/10.1016/0022-1694(81)90078-0

Holden, H., Lindstrøm, T., Øksendal, B., Ubøe, J., \& Zhang, T.-S. (1993). Stochastic boundary value problems: a white noise functional approach. Probability Theory and Related Fields, 95(3), 391-419. doi:10.1007/bf01192171

Holden, H., Oksendal, B., Uboe, J., \& Zhang, T. (1996). Stochastic Partial Differential Equations: A Modeling, White Noise Functional Approach (1 ed.): Birkhäuser Basel.

Kalpinelli, E. A., Frangos, N. E., \& Yannacopoulos, A. N. (2011). A Wiener Chaos Approach to Hyperbolic SPDEs. Stochastic Analysis and Applications, 29(2), 237-258. doi:10.1080/07362994.2011.532023

Kondratiev, Y., Leukert, P., \& Streit, L. (1996). Wick calculus in Gaussian analysis (Vol. 44). Kulasiri, D. (2013). Non-fickian Solute Transport in Porous Media. Berlin, Heidelberg: Springer.

Luo, W. (2006). Wiener chaos expansion and numerical solutions of stochastic partial differential equations. . (Dissertation (Ph.D.)), California Institute of Technology. Retrieved from http://resolver.caltech.edu/CaltechETD:etd-05182006-173710

Natarajan, N., \& Kumar, G. S. (2018). Spatial moment analysis of multispecies contaminant transport in porous media. Environmental Engineering Research, 23(1), 76-83. doi:10.4491/eer.2016.147

Peterson, T. J., \& Western, A. W. (2014). Nonlinear time-series modeling of unconfined groundwater head. Water Resources Research, 50(10), 8330-8355. doi:10.1002/2013wr014800

Serrano, S., \& Unny, T. (1987). Stochastic Partial Differential Equations in Hydrology.

Tegegne, G., Kim, Y.-O., Seo, S. B., \& Kim, Y. (2019). Hydrological modelling uncertainty analysis for different flow quantiles: a case study in two hydro-geographically different watersheds. Hydrological Sciences Journal, 64(4), 473-489. doi:10.1080/02626667.2019.1587562

Xu, H., Cantwell, C. D., Monteserin, C., Eskilsson, C., Engsig-Karup, A. P., \& Sherwin, S. J. (2018). Spectral/hp element methods: Recent developments, applications, and perspectives. Journal of Hydrodynamics, 30(1), 1-22. doi:10.1007/s42241-018-0001-1

Ye, T., \& Zhu, Y. (2018). A METRIC ON UNCERTAIN VARIABLES. 8(3), 251-266. doi:10.1615/Int.J.UncertaintyQuantification.2018020455

Zhang, Z., Rozovskii, B., Tretyakov, M. V., \& Karniadakis, G. E. (2012). A Multistage Wiener Chaos Expansion Method for Stochastic Advection-Diffusion-Reaction Equations. SIAM Journal on Scientific Computing, 34(2), A914-A936. doi:10.1137/110849572

Zhang, Z. a. (2017). Numerical methods for stochastic partial differential equations with white noise: Cham, Switzerland : Springer, [2017]. (C2017. 